\title{
CARACTERIZAÇÃO DOS EXTREMOS DE PRECIPITAÇÃO DIÁRIA NO NORDESTE DO BRASIL
}

\author{
CHARACTERIZATION OF DAILY PRECIPITATION \\ EXTREMES ON NORTHEASTERN BRAZIL
}

\author{
CARACTERÍZACION DE LOS EXTREMOS DE PRECIPITACIÓN \\ DIARIA EN EL NORESTE DE BRASIL
}

\author{
Washington Luiz Félix Correia Filho - Universidade Federal do Rio \\ Grande do Norte - Natal - Rio Grande do Norte - Brasil \\ wlfcfm@hotmail.com
}

\begin{abstract}
Paulo Sérgio Lucio - Universidade Federal do Rio Grande do Norte - Rio Grande do Norte - Natal - Brasil
pslucio@ccet.ufrn.br
\end{abstract}
Maria Helena Constantino Spyrides - Universidade Federal do Rio Grande do Norte - Natal -Brasil spyrides@ccet.ufrn.br

\begin{abstract}
Resumo
A intensificação das mudanças climáticas tornou os eventos extremos mais frequentes. No Nordeste do Brasil (NEB) estes eventos estão associados às secas ou enchentes. Neste trabalho utilizaram-se as distribuições generalizadas de valores extremos (GEV) e de Pareto (GPD), com o objetivo de caracterizar os máximos de precipitação no NEB e avaliar os níveis e períodos de retorno para 30,50 e 100 anos, baseado nos meses de fevereiro a julho do período de 1979-2011. Os resultados do parâmetro de localização e de escala apontaram que ambas as distribuições apresentaram padrões espaciais similares, os máximos de precipitação localizados na costa leste do NEB, no norte do Maranhão e do Ceará; e um núcleo no extremo oeste de Pernambuco (influenciado pela topografia do planalto da Borborema e da chapada do Araripe). 0 parâmetro de forma mostrou que as regiões selecionadas se ajustaram pelas distribuições de Weibull negativa e beta $(\xi<0)$ para GEV e GPD, respectivamente. As estimativas dos níveis e períodos de retorno da GEV (GPD) mostraram que na região norte do Maranhão (região central da Bahia) ocorrerá pelo menos um evento extremo de precipitação no nível igual ou superior a 160,9 mm/dia (192,3 mm/dia) em 30 anos, e de 182,1mm/dia (307,8mm/dia) em 100 anos.
\end{abstract}

Palavras-chave: planalto da borborema, norte do Maranhão, distribuição GEV.

\section{Abstract}

The intensification of climate change impacts made the extreme events more frequent. On Northeastern Brazil (NEB) these events are closely associated to drought or floods. In this work, the generalized extreme value (GEV) and Pareto (GPD) distributions were used with the objective to characterize the precipitation extremes on NEB and to assess the levels and return periods for 30,50 and 100 years, based on the period of February to July from 1979 to 2011. The location and scale parameters results pointed that both distributions showed similar spatial patterns, the maximum precipitation located on the east coast of the NEB, on northern Maranhão and Ceará States; and a core in the extreme western region of Pernambuco State (influenced by topography of the Borborema and Araripe plateaus). The shape parameter showed that selected regions are set by Weibull negative and Beta distributions $(\xi<0)$ for GEV and GPD, respectively. The estimate of levels 
and return periods by GEV distribution showed that on Northern Maranhão State (central Bahia region) will occur at least one extreme precipitation event with equal or superior level to $160,9 \mathrm{~mm} /$ day $(192,3 \mathrm{~mm} /$ day $)$ in 30 years, and $182,1 \mathrm{~mm} /$ day $(307,8 \mathrm{~mm} /$ day $)$ on the next 100 years.

Keywords: borborema plateau, northern Maranhão State, GEV distribution.

\section{Resumen}

La intensificación de los cambios climáticos ha hecho que los eventos extremos sean más frecuentes. En el noreste de Brasil (NEB), estos eventos están estrechamente asociados a sequías o inundaciones. En este trabajo se utilizaron las distribuciones generalizadas de valores extremos (GVE) y de Pareto (GPD) con el fin de caracterizar los máximos de precipitación en el NEB y evaluar los niveles y períodos de retorno para 30,50 y 100 años, sobre la base de los meses de febrero a julio del periodo 1979 a 2011. Los resultados del parámetro de localización y de escala mostraron que ambas distribuciones presentan patrones espaciales similares, los máximos localizados en la costa este de la NEB, en el norte de Maranhão y Ceará y un núcleo en el extremo oeste de Pernambuco (influenciado por la topografía de la meseta Borborema y Araripe). El parámetro de forma mostró que las regiones seleccionadas se ajustaron por las distribuiciones de Weibull negativa y beta $(\xi<0)$ para GEV y GPD, respectivamente. Las previsiones de los niveles y períodos de retorno del GEV (GPD) mostraron que en la región norte de Maranhão (región cenral de Bahía) habrá al menos un evento de precipitación extrema de nivel igual o superior a 160,9 mm/día (192,3 mm/día) en los próximos 30 años y de $182,1 \mathrm{~mm} /$ día (307,8 mm/día) en los próximos 100 años.

Palabras Clave: Meseta de la Borborema, norte de Maranhão, distribución GEV.

\section{Introdução}

O aumento progressivo no número de eventos extremos ao longo do mundo têm preocupado a comunidade internacional, devido à magnitude e dimensão de seus impactos, resultando em enormes prejuízos socioeconômicos e ambientais, afetando aqueles que são mais vulneráveis (Seneviratne et al., 2012; Mishra et al., 2015).

Estes eventos extremos são desencadeados pela ação antrópica ao meio ambiente, isoladamente ou associados ao fatores ambiental ou regional, ao qual pode ser classificados como: hidrológico, climatológico, geológico e meteorológico (Freitas et al., 2014). Estes eventos podem ocorrer motivados por: alterações nos padrões dos sistemas meteorológicos em várias escalas espaço e de tempo (Meehl; Tebaldi, 2004; Cavalcanti, 2012), alterações na variáveis meteorológicas (Kayano et al., 2013; Correia-Filho et al., 2014), influência topográfica (Houze, 2012) ou microclima regional (Willems et al., 2012).

Alguns destes elementos estão interligados as mudanças climáticas, verificada pelos padrões anômalos das variáveis termodinâmicas (temperatura e capacidade de armazenamento de água da atmosfera) e dinâmicas (padrão de circulação dos ventos), contribuíndo para o aumento na frequência dos eventos extremos e de seus impactos (Trenberth, 2012; Cavalcanti, 2012). 
De acordo com Trenberth (2012), estes extremos devem ser avaliados de forma individual, pois em sua maioria não apresentam características similares entre eles, ao qual recorre-se a análise dos conglomerados nos extremos para a identificação de padrão anômalo da variabilidade natural.

Os eventos extremos que ocorrem no Brasil são influenciados por sistemas meteorológicos, de forma isolada ou combinada atuam de forma regional ou global, geralmente estão associados a chuvas extremas, inundações ou secas (Cavalcanti, 2012; Marengo et al., 2013; Marengo et al., 2016).

No Nordeste do Brasil (NEB), estes extremos estão diretamente associados a precipitação pluvial. Esses sistemas exercem uma forte influência no regime pluviométrico no NEB, principalmente em seus extremos, um destes é a variabilidade da Temperatura à Superfície do Mar (TSM) do Atlântico e Pacífico tropicais (Andreoli; Kayano, 2006; Kayano et al., 2013), ao qual as fases anômalas de cada um dos oceanos podem atuar em separado ou em conjunto. Há outros sistemas meteorológicos atuantes que favorecem a ocorrência de chuvas extremas no NEB, por exemplo, a Zona de Convergência Interotropical (ZCIT) e os Distúrbios Ondulatórios de Leste (DOL) (Torres; Ferreira, 2011).

Quando trata-se de escassez de chuvas, logo associa-se a seca do semiárido nordestino, localizado na parte central do NEB, região que vivencia longos períodos de estiagem, ocasionado pela forte irregularidade no regime de chuvas e de sua má distribuição espacial (Marengo et al., 2016).

As secas no NEB impactam fortemente nas condições socioambientais e econômicas na região, gerando enormes prejuízos no setor agropecuário, principalmente para a população que sobrevivem da agricultura de subsistência, de maneira mais severa em anos sob vigência do El Niño-Oscilação Sul (Araújo et al., 2013; Kayano et al. 2013; Sousa; Souza, 2014; Silva et al., 2016).

Em contrapartida, quando se aborda as chuvas extremas no NEB, ideia central deste trabalho, são comumente verificadas em regiões litorâneas, inclusive na maioria das capitais nordestinas. Este tipo de evento, quando associado a cidades com infraestrutura precária (sistemas de saneamento básico e de drenagem ineficientes) combinada ao crescimento desordenado sem planejamento das cidades, resulta em alagamentos e inundações (Freire et al., 2013; Queiroz Rosendo et al., 2015). 
A ocorrência de chuvas extremas em cidades de médio e grande porte pode desencadear uma série de problemas, um destes é de saúde pública, pois os alagamentos ou inundações provocados pela chuva contribuem para o surgimento de surtos endêmicos, pois o contato com água contaminada pelo lixo, ou pela urina de roedores, ou vetores podem resultar em transmissão de diversas doenças (Confalonieri, 2003; Rufino et al., 2016). Além disso, a falta de infraestrutura pode limitar alguns serviços elementares à população, tais como: o fornecimento de energia elétrica e abastecimento de água, restrição da mobilidade urbana (Amorim et al., 2014).

Para analisar a ocorrência destes chuvas extremas, diversas metodologias vêm sendo aplicadas e utilizadas, duas das mais conhecidas são: 1) índices climáticos, baseada em índices de extremos climáticos criados pela World Meteorological Organization (2009), com o propósito de verificar a tendência e sazonalidade dos extremos; 2) Teoria dos Valores Extremos (TVE) (Scarrot; MacDonald, 2012), baseadas nas distribuições generalizadas de valores extremos (GEV) e de Pareto (GPD), que são extraídos a localização, escala e forma destes eventos.

A TVE é comumente aplicada em dados de temperatura e de precipitação, devido à sensibilidade de ambas para qualquer alteração sutil no clima pode ser facilmente perceptível por ambas as variáveis (Scarrot; MacDonald, 2012).

A partir da TVE para caracterizar a ocorrência de chuvas extremas, o objetivo deste trabalho será captar sinais dos extremos de precipitação no NEB, a partir das distribuições GEV e GPD com o percentil 95, avaliando o comportamento dos níveis e períodos de retorno de 30, 50 e 100 anos, utilizando dados diários de 1979-2011 para o período de fevereiro a julho (período chuvoso).

Material e métodos

\section{Dados}

Neste trabalho serão utilizados dados diários de precipitação pluvial, disponibilizados pelo Climate Prediction Center - National Oceanic and Atmospheric Administration (CPC-NOAA) (Joyce et al., 2004; Chen et al., 2008), com resolução de grade de $0,5^{\circ} \times 0,5^{\circ}$ do período de fevereiro 
a julho de $1979-2011$, e redimensionados para $1,5^{\circ} \times 1,5^{\circ}$, totalizando sete e sete pontos de grade, ao qual cobrem todo o Nordeste, abrangendo uma área entre as latitudes $0-18^{\circ} \mathrm{S}$ e longitudes $35-48^{\circ} \mathrm{W}$, vista na Figura 1.

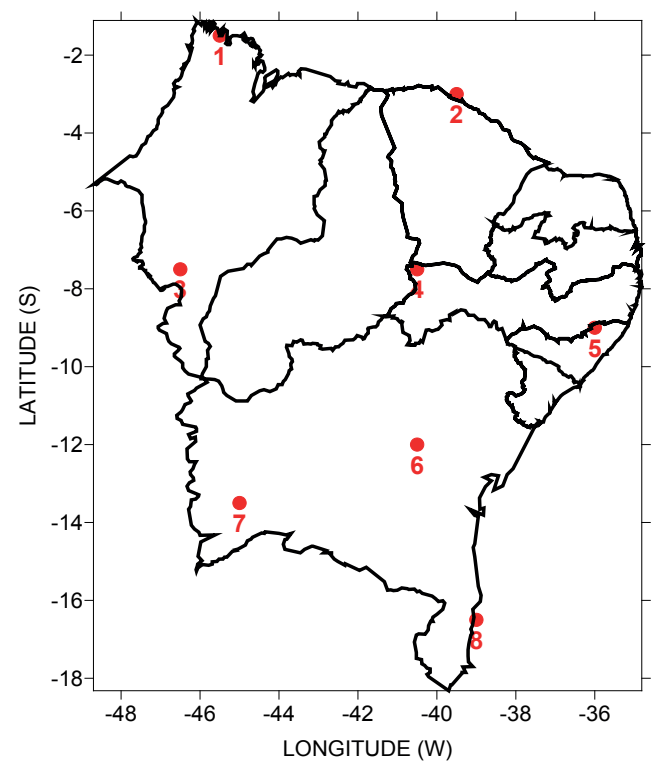

Figura 1 - Localização dos pontos das oito regiões selecionadas para avaliação do extremos de precipitação diária no Nordeste do Brasil Fonte: Elaborado pelos autores.

Para avaliar o comportamento destes eventos ao longo da Região Nordeste do Brasil, analisou-se oito regiões, avaliando comportamentos distintos: litoral e costeira, composto pelas regiões 1 (norte do Maranhão, NMA), 2 (norte do Ceará, NCE), 5 (nordeste de Alagoas, NAL) e 8 (sul da Bahia, SBA); semiárido, composto pelas regióes 4 (oeste de Pernambuco, OPE), 6 (região central da Bahia, CBA) e 7 (sul da Bahia, SBA); e região de transição entre amazônia e semiárido, composto pela região 3 (sul do Maranhão, SMA). Nesta sub-seção abordaremos a aplicação da TVE baseada nas distribuições GEV e GPD, encontrada com maiores detalhes em Coles et al. (2001). 


\section{Distribuição Generalizada de Valores Extremos (GEV)}

Este método consiste em extrair de uma série de observações independentes $X_{t}, t=1 \ldots \mathrm{n}$, um valor máximo a partir de um intervalo de observações $n$, gerando uma série de máximos extraída destes blocos, que podem ser diário, mensal, anual. A função de distribuição acumulada da distribuição GEV é dada por:

$$
F(z)=\exp \left\{-\left[1+\xi\left(\frac{x-\mu}{\sigma}\right)\right]^{-1 / \xi}\right\}, \quad z: 1+\xi(x-\mu) / \sigma>0
$$

Em que $\mu, \sigma$ e $\xi$ são os parâmetros de localização, de escala e de forma, respectivamente. Em caso particular, quando $\xi \rightarrow 0$ (Distribuição Gumbel), tem-se:

$$
G(z)=\exp \left\{-\exp \left(-\frac{x-\mu}{\sigma}\right)\right\}, \quad-\infty<x<\infty
$$

A obtenção do período de retorno pela seguinte forma:

$$
x_{T}=\mu-\frac{\sigma}{\xi}\left[1-\left\{-\log \left(1-\frac{1}{T}\right)\right\}^{-\xi}\right]
$$

$x_{T}$ é o nível de retorno de um evento extremo de precipitação ocorra em um dia dado um período de $T$ anos.

\section{Distribuição Generalizada de Pareto (GPD)}

A distribuição Generalizada de Pareto (GPD) consiste em estabelecer um critério padrão ao conjunto de dados, sendo estes horários, diários ou mensais, sem que haja qualquer necessidade de formar blocos. Seja $X_{1}, \ldots, X_{n}$, uma série de variáveis aleatórias independentes e identicamente distribuídas (iid), que contenha uma função de distribuição $\mathrm{F}(\mathrm{x})$ desconhecida, sendo $\mathrm{X}_{\mathrm{i}}$ as observações de valores diários de precipitação, o principal interesse é estimar o comportamento da precipitação acima do maior limiar m (threshold) (Coles et al., 2001).

Na distribuição GPD, aplicou-se o método dos picos aleatórios acima dos limiares ("Peaks Over Random Threshold" - PORT) nas séries temporais de precipitação para cada ponto de grade da região de estudo (Scarrot; MacDonald, 2012). 
De acordo com Folland et al. (1999), a utilização de limiares absolutos flexíveis baseados em percentis são recomendados para regiões que apresentem características fisiográficas ou climáticas heterogêneas, evitando-se assim mascarar o resultado final, neste trabalho utilizou-se o percentil 95. Para estimar a distribuição de excessos utilizamos a Equação:

$$
F_{u}(y)=P\{X>u+y \mid X>u\}=\frac{F(u+y)-F(u)}{1-F(u)}
$$

Em que $0 \leq y<r_{F}-u, r_{F}=\inf (x: F(x)=1) \leq \infty$, com o limite à direita $\mathrm{F}(\mathrm{x})$.

A distribuição dos excessos representa a probabilidade em que um evento extremo de precipitação $\mathrm{X}_{\mathrm{i}}$ exceda um limiar $\mu$, ou seja, quando a GPD é parametrizada pelos parâmetros de escala e forma nos termos dos limiares excedentes $x-\mu$, assim, representando os excedentes $x>\mu$, podemos representar a GPD desta maneira:

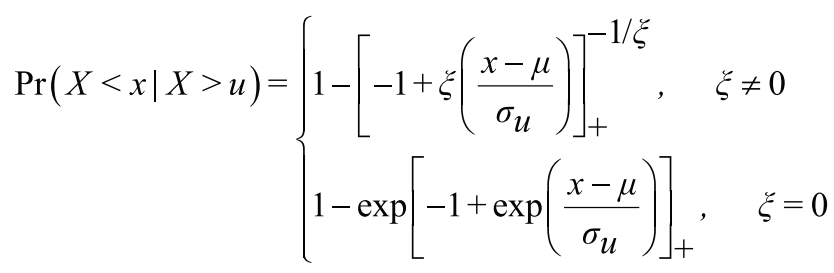

Em que $\mathrm{y}_{+}=\max (\mathrm{y}, 0)$. Quando o $\xi<0$ no lado superior, então $\mu<\mathrm{x}<\mu$-.

De forma implícita a estimação do nível de retorno é proporcional aos limiares excedentes, assim o período de retorno é obtido pela seguinte Equação:

$$
x_{N}=u(\mathrm{t})+\frac{\sigma(\mathrm{t})}{\xi}\left[\left(\mathrm{Nn} \zeta_{u} \theta\right)^{\xi}-1\right]
$$

Cada um dos três parâmetros das distribuições GEV e GPD exibem características distintas a partir da série de extremos: 1) localização $(\mu)$, avalia o comportamento médio dos extremos de precipitação; 2) escala ( $\sigma$ ), verifica os desvios dos valores dos extremos; e 3) forma ( $\xi$ ), indica qual distribuição a série utilizada se ajusta, este ajuste é determinante para identificar se estes extremos serão mais frequentes e intensos $(\xi>0)$ ou ocorrerá o contrário $(\xi<0)$. 


\section{Resultados e discussão}

Os resultados discutidos a seguir referem-se aos parâmetros de localização, escala e de forma das distribuições GEV (Figuras 2a, 3a e 4a, respectivamente) e GPD (Figuras $2 b$, $3 b$ e $4 b$, respectivamente), com base nos máximos anuais de precipitação sobre o NEB.

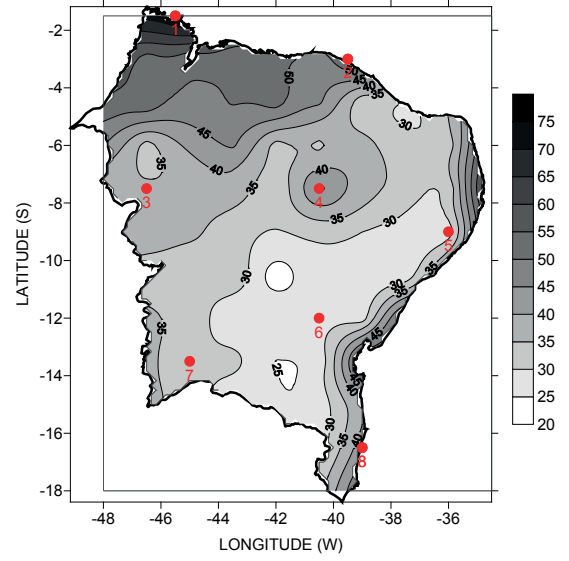

(A)

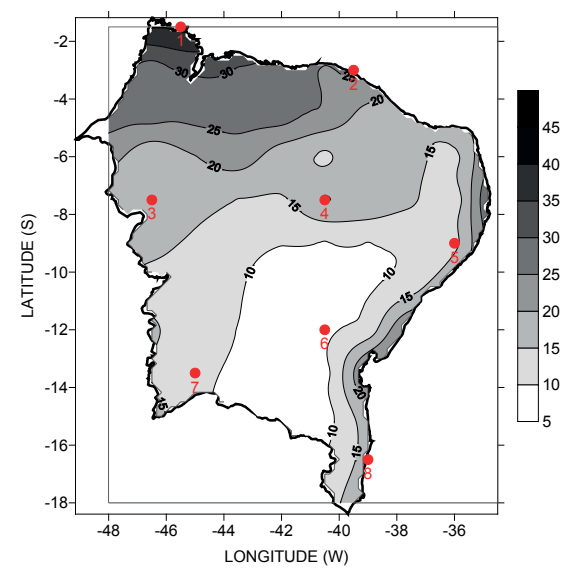

(B)

Figura 2 - Diagnóstico dos extremos de precipitação no NEB a partir do parâmetro de localização $(\mu$, em mm) das distribuições: (A) GEV e (B) GPD. A legenda é dada em mm.

Fonte: Elaborado pelo autor.

Os parâmetros de localização ( $\mu$ ) (Figuras 2a e 2b) apresentam padrões similares em ambas distribuições, com três regiões de máxima: $1^{\text {a }}$ ) se estende ao longo da costa leste do NEB, do extremo leste do Rio Grande do Norte ao sul da Bahia; $2^{\mathrm{a}}$ ) nas regiões NMA e NCE; e $3^{\mathrm{a}}$ ) na região OPE.

Os máximos de precipitação observados na região NMA variam entre 77,6 mm e $42 \mathrm{~mm}$ para as distribuições GEV e GPD (Tabela 1), respectivamente, enquanto o menor foi verificado na região NAL, com valores entre $26,7 \mathrm{~mm}$ e $13,3 \mathrm{~mm}$, respectivamente.

As regiões com os maiores valores do parâmetro de localização ( $\mu$ ) (contidos na Tabela 1) estão influenciadas por dois fatores, o primeiro baseado em dois sistemas meteorológicos atuantes no NEB, a ZCIT (Souza; Cavalcanti, 2009) na parte norte, que possui um papel fundamental no regime pluviométrico na região, e de seus extremos, motivados pelo 
deslocamento mais ao sul da climatologia entre as latitudes $1^{\circ} \mathrm{S}-3^{\circ} \mathrm{S}$, impulsionando as atividade convectivas sobre a região, propiciando a ocorrência destes extremos.

Tabela 1 - Parâmetros de $\mu, \sigma$ e $\xi$ das distribuições GEV e GPD para as oito regiões selecionadas: NMA, NCE, SMA, OPE, NAL, CBA, OBA e SBA

\begin{tabular}{|c|c|c|c|c|c|c|}
\hline & \multicolumn{3}{|c|}{ GEV } & \multicolumn{3}{c|}{ GPD } \\
\hline Localização & $\mu(\mathrm{mm})$ & $\sigma(\mathrm{mm})$ & $\xi$ & $\mu(\mathrm{mm})$ & $\sigma(\mathrm{mm})$ & $\xi$ \\
\hline NMA (região1) & 77,6 & 31,2 & $-0,14$ & 42,0 & 17,4 & 0,11 \\
\hline NCE (região 2) & 59,0 & 28,5 & $-0,16$ & 27,1 & 15,6 & 0,08 \\
\hline SMA (região 3) & 35,6 & 15,2 & $-0,32$ & 16,8 & 9,62 & $-0,00$ \\
\hline OPE (região 4) & 46,5 & 19,4 & $-0,17$ & 20,8 & 11,8 & 0,07 \\
\hline NAL (região 5) & 26,7 & 7,8 & 0,27 & 13,3 & 6,60 & 0,17 \\
\hline CBA (região 6) & 28,3 & 15,0 & 0,03 & 9,2 & 7,10 & 0,37 \\
\hline OBA (região 7) & 31,0 & 12,8 & $-0,26$ & 11,5 & 8,84 & 0,03 \\
\hline SBA (região 8) & 43,0 & 14,8 & $-0,54$ & 17,1 & 11,66 & $-0,04$ \\
\hline
\end{tabular}

Fonte: Elaborado pelo autor.

O segundo sistema é o DOL na parte leste (região costeira), motivados pela propagação de ondas advindas da África, que transportam calor e umidade do Oceano Atlântico Tropical para a região costeira, resultando em chuvas extremas (Kouadio et al., 2012), no entanto, ambos estão interligados à variabilidade da TSM do Oceano Atlântico Tropical (Andreoli; Kayano, 2006).

O segundo fator é a influência orográfica, verificado na região OPE, situada no planalto da Borborema e da chapada do Araripe, localizada entre os estados de Pernambuco, Paraíba, Rio Grande do Norte e Ceará, também conhecida como Planalto Nordestino. A elevação deste planalto varia entre 200-1000 m de altitude, exerce uma forte interferência no regime pluviométrico na região, e em consequência, em seus extremos.

Os resultados apresentados pela distribuição GEV apontam similaridade aos resultados encontrados por Easterling et al. (2000). Na ocasião, os autores concluíram que a média diária dos extremos de precipitação no NEB é de 50,8 mm/dia, no entanto, na parte norte do NEB esse valor é facilmente atingido, na região SMA já foram registrados casos de extremos de precipitação diários com valores superiores a 100 
$\mathrm{mm} /$ dia. Com base no período de estudo, o máximo registrado ocorreu em 2008, com o valor de 168,5 mm.

O resultado do parâmetro de escala $(\mu)$ (visto na Tabela 1 e Figura 2) aponta as regiões propensas a sofrerem com a ocorrência de extremos de chuva, ao qual alerta sobre a necessidade de ações do governo para a prevenção de desastres, para que se tome medidas para minimizar os impactos causados, o grande problema é que parte da população vive em regiões de risco, de relevo acidentado ou próximo locais de escoamento das águas superficiais / pluviais, aumentando assim sua vulnerabilidade.

Avaliando o parâmetro de escala $(\sigma)$ para as distribuições GEV (Figura 3a) e GPD (Figura 3b), a região do semiárido apresenta menor variabilidade, com valores inferiores a $10 \mathrm{~mm}$. Quanto aos valores deste parâmetro (Tabela 1), os valores variam entre 17,4 mm (região NAL) e 31,2 mm (região NMA) para a GEV, e entre 6,6 mm (região NAL) a 7,8 mm (região NCE) para GPD.

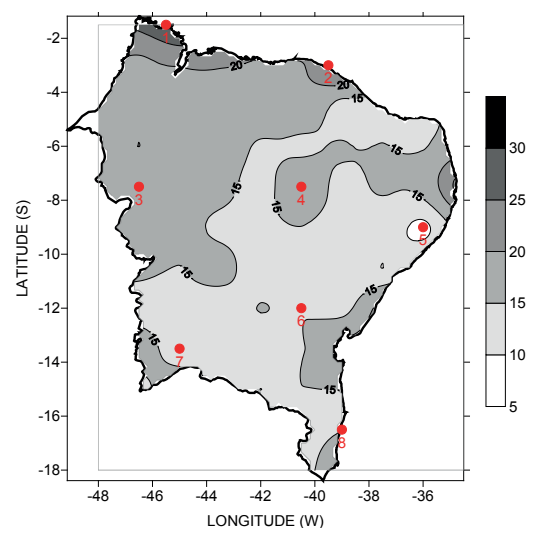

(A)

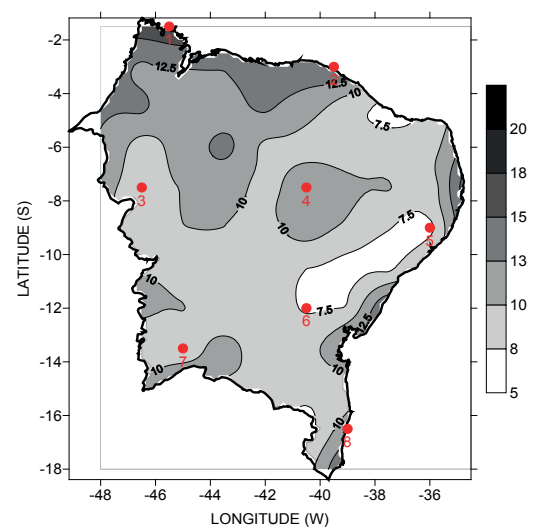

(B)

Figura 3 - Diagnóstico dos extremos de precipitação no NEB a partir do parâmetro de escala $(\sigma$, em $\mathrm{mm}$ ) das distribuições: (A) GEV e (B) GPD. A legenda é dada em mm Fonte: Elaborado pelo autor.

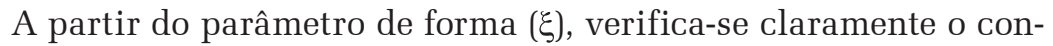
traste entre os resultados gerados pela GEV (Figura 4a) e GPD (Figura 4b). Pela GEV, as regiões NAL e CBA são ajustadas pela distribuição Fréchet $(\xi>0)$ e Gumbel $(\xi=0)$, respectivamente, enquanto as demais foram ajustadas pela distribuição Weibull negativa $(\xi<0)$. 


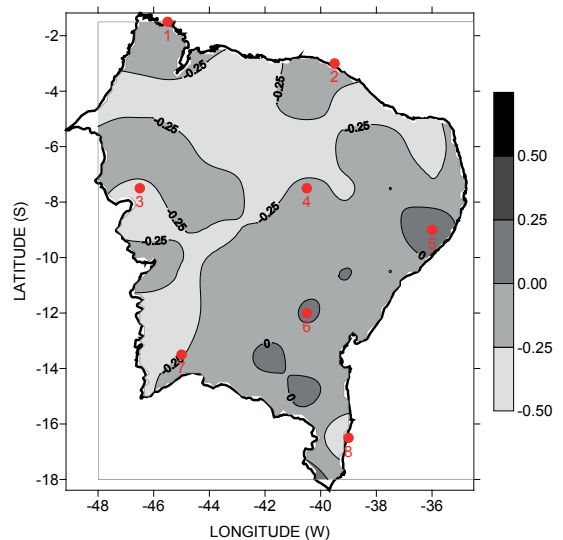

(A)

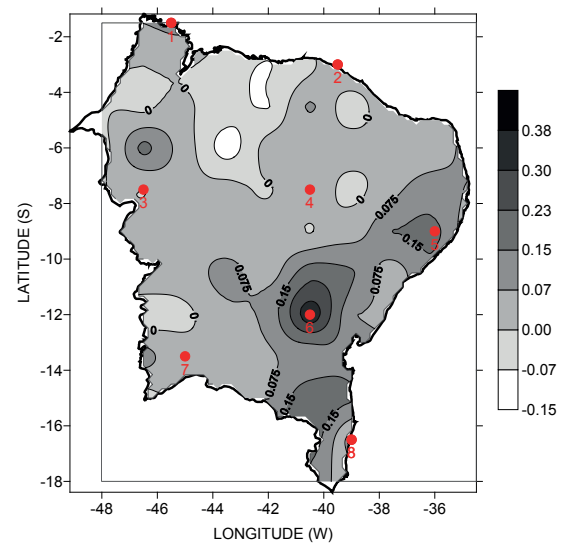

(B)

Figura 4 - Diagnóstico dos extremos de precipitação no NEB a partir do parâmetros de forma $(\xi)$ da distribuição: (A) GEV e (B) GPD. A legenda é adimensional Fonte: Elaborado pelo autor.

No caso da GPD, as regiões SMA e SBA são ajustadas pelas distribuições exponencial $(\xi=0)$ e beta $(\xi<0)$, respectivamente, enquanto as demais são ajustadas pela distribuição de Pareto $(\xi>0)$.

A verificação do ajuste dos dados é vital para a obtenção dos níveis e períodos de retorno estimados para 30, 50 e 100 anos, quando o parâmetro de forma é negativo o período de retorno aumenta enquanto o nível atenua até ser constante, quando o parâmetro de forma é positivo ocorre o contrário.

A partir da estimação dos níveis e períodos de retornos para as distribuições GEV e GPD (Tabela 2), constatamos que os maiores níveis de retorno foram verificados nas regiões NMA para GEV e NCE para GPD, os menores níveis foram observados na região OBA para ambas as distribuições, para a maioria das regiões selecionadas os níveis são maiores na GPD do que na GEV.

Tabela 2 - Níveis (mm/dia) e períodos de retornos estimados para 30, 50 e 100 anos via distribuições GEV e GPD

\begin{tabular}{|c|c|c|c|c|c|c|}
\hline \multirow{2}{*}{} & \multicolumn{3}{|c|}{ GEV } & \multicolumn{3}{c|}{ GPD } \\
\cline { 2 - 7 } & \multicolumn{3}{|c|}{ Período (em anos) } & \multicolumn{3}{c|}{ Período (em anos) } \\
\hline Localização & 30 & 50 & 100 & 30 & 50 & 100 \\
\hline NMA (região 1) & 160,9 & 170,3 & 182,1 & 88,6 & 99,1 & 115,0 \\
\hline
\end{tabular}




\begin{tabular}{|c|c|c|c|c|c|c|}
\hline NCE (região 2) & 133,4 & 141,6 & 151,7 & 158,4 & 172,6 & 192,8 \\
\hline SMA (região 3) & 67,1 & 69,6 & 72,3 & 76,4 & 81,1 & 87,6 \\
\hline OPE (região 4) & 96,3 & 101,6 & 108,1 & 113,8 & 123,2 & 136,5 \\
\hline NAL (região 5) & 70,7 & 81,8 & 99,7 & 88,6 & 99,1 & 114,9 \\
\hline CBA (região 6) & 82,3 & 91,1 & 103,2 & 192,3 & 235,0 & 307,8 \\
\hline OBA (região 7) & 59,7 & 62,2 & 65,2 & 72,4 & 77,8 & 85,3 \\
\hline SBA (região 8) & 66,1 & 67,2 & 68,3 & 81,1 & 85,5 & 91,4 \\
\hline
\end{tabular}

Fonte: Elaborado pelo autor.

Sobre o nível de retorno obtido pela GEV, constata-se que poderá ocorrer pelo menos um evento extremo de precipitação de nível igual ou superior a 160,9 mm/dia em 30 anos, e de 182,1 mm/dia em 100 anos. Enquanto isso nas regiões SMA, OBA e SBA haverá pequenas alterações em seus extremos de precipitação.

A distribuição GPD exibe comportamento distinto quando comparada a GEV, o maior nível de retorno ocorre na região CBA e poderá ocorrer pelo menos um evento extremo de precipitação do nível igual ou superior a 192,3 mm/dia nos próximos 30 anos, e um de 307,8 mm/dia nos próximos 100 anos.

\section{Conclusões}

A partir dos resultados apresentados, obteve-se algumas conclusões: os máximos se concentraram nas regiões litorâneas (norte do Maranhão, do Ceará e na costa leste do NEB) influenciadas pelos sistemas atmosféricos que atuam neste período (ZCIT e DOL), já a região OPE é influenciada pela topografia (motivada pelo planalto da Borborema e chapada do Araripe).

As distribuições GEV e GPD apresentaram diferenças significativas entre elas, vistos na intensidade dos extremos e na distribuição espacial do parâmetro de forma, com os maiores valores na distribuição GEV. No entanto, as estimativas dos níveis e períodos de retorno foram superiores na GPD quando comparados a GEV, com o maior valor na regiões NMA (NCE) para GEV (GPD).

Os parâmetros de localização e de escala apresentaram similaridades nos aspectos da região de influência e em suas intensidades (valores de máximas e mínimas). No parâmetro de forma, a maioria das séries selecionadas foi ajustada pela distribuição de Weibull negativa e beta $(\xi<0)$ para GEV e GPD, respectivamente. Os valores dos parâmetros para cada 
distribuição foram: de localização, entre 26,7 a 77,6 mm para GEV e 9,2 a 42,0 $\mathrm{mm}$ para GPD; de escala, entre 7,8-31,2 $\mathrm{mm}$ para GEV e 6,6 a 17,4 mm para GPD; e de forma, entre -0,54 a 0,27 para GEV e -0,04 a 0,37 para GPD. Pela GEV, conclui-se que a região NMA pode notificar pelo menos um evento extremo de precipitação de nível igual ou superior a 160,9 $\mathrm{mm} /$ dia nos próximos 30 anos, e um de 182,1 mm/dia nos próximos 100 anos, enquanto pela GPD, na região CBA pode notificar pelo menos um evento extremo de precipitação de nível igual ou superior a 192,3 mm/dia nos próximos 30 anos e um de 307,8 mm/dia para os próximos 100 anos.

\section{Agradecimentos}

O autor agradece a Coordenação de Aperfeiçoamento de Pessoal Nível Superior (CAPES) pela concessão da Bolsa de pós-doutoramendo pelo PNPD. O presente trabalho é parte dos resultados obtidos da Tese de Doutorado financiado pela CAPES - Brasil, dentre os anos de 2010-2014. P. S. Lucio é bolsista PQ2 do CNPq (Proc: 309165/2010-5). Aos revisores anônimos que contribuíram com recomendações e sugestões para a melhoramento deste trabalho.

\section{Referências}

AMORIM, M. F. de.; QUELHAS, O. L. G.; MOTTA, A. L. T. S. da. The resilience of cities across the torrential rains: case study of the contingency plan of the city of Rio de Janeiro (Brazil). Sociedade \& Natureza, v. 26, n. 3, p. 519-534, 2014.

ANDREOLI, R. V.; KAYANO, M. T. Tropical Pacific and South Atlantic effects on precipitation variability over Northeastern Brazil. International Journal of Climatology, , v. 26, n.13, p. 1895-1912, 2006. DOI. 10.1002/JOC.1341.

ARAÚJO, P. H. C. et al. Eventos climáticos extremos: efeitos dos fenômenos El Niño e La Niña sobre a produtividade agrícola. In: BOUERI, R.; COSTA, M. A. (Org.). Brasil em desenvolvimento 2013: estado, planejamento e políticas públicas. Brasília: Ipea, 2013. p. 347-362.

CAVALCANTI, I. F. A. Large scale and synoptic features associated with extreme precipitation over South America: a review and case studies for the first decade of the 21st century. Atmospheric Research, v. 118, p. 27-40, 2012.

CHEN, M. et al. Assessing objective techniques for gauge-based analyses of global daily precipitation. Journal of Geophysical Research, v. 113 (D4), D04110, 2008.

COLES, S. et al. An introduction to statistical modeling of extreme values. London: Springer, 2001. $209 \mathrm{p}$. 
CONFALONIERI, U. E. C. Variabilidade climática, vulnerabilidade social e saúde no Brasil. Fundação Oswaldo Cruz, Terra Livre, São Paulo, ano 19, v. I, n. 20, p. 193-204, 2003.

CORREIA-FILHO, W. L. F.; LUCIO, P. S.; SPYRIDES, M. H. C. Precipitation extremes analysis over the Brazilian Northeast via logistic regression. Atmospheric and Climate Science, v. 4, n. 1, p. 53-59, 2014.

EASTERLING, D. R. et al. Observed variability and trends in extreme climate events: a brief review. Bulletin of the American Meteorological Society, v. 81, n. 3, p. 417-425, 2000.

FOLLAND, C. K. et al. Workshop on indices and indicators for climate extremes, Asheville, NC, USA, 3-6 June 1997 Breakout Group C: temperature indices for climate extremes. Climatic Change, v. 42, n. 1, p. 31-43, 1999.

FREIRE, F. G. C. et al. Estudo das precipitações máximas para o município de Mossoró-RN, Brasil. Revista Brasileira de Agricultura Irrigada-Rbai, v. 6, n.1, p. 3-7, 2013.

FREITAS, C. M. et al. Desastres naturais e saúde: uma análise da situação do Brasil. Ciência \& Saúde Coletiva, v. 19, n. 9, p. 3645-3656, 2014.

HOUZE, R. A. Jr. Orographic effects on precipitating clouds. Review of Geophysics, v. 50 RG1001, 2012. DOI:10.1029/2011RG000365.

JOYCE, R. J. et al. A method that produces global precipitation estimates from passive microwave and infrared data at high spatial and temporal resolution. Journal of Hydrometeorology, v. 5, n. 3, p. 487-503, 2004.

KAYANO, M. T.; ANDREOLI, R. V.; SOUZA, R. A. F. Relations between ENSO and the South Atlantic SST modes and their effects on the South American rainfall. International Journal of Climatology, v. 33, n. 8, p. 2008-2023, 2013.

KOUADIO, Y. K. et al. Heavy rainfall episodes in the eastern northeast brazil linked to large-scale ocean-atmosphere conditions in the tropical atlantic. Advances in Meteorology, 2012.

MARENGO, J. A. et al. Two contrasting severe seasonal extremes in tropical South America in 2012: flood in Amazonia and drought in northeast Brazil. Journal of climate, v.26, n. 22, p. 9137-9154, 2013.

MARENGO, J. A.; TORRES, R. R.; ALVES, L. M. Drought in Northeast Brazil - past, present, and future. Theoretical and Applied Climatology, p. 1-12, 2016.

MEEHL, G. A.; TEBALDI, C. More intense, more frequent, and longer last- ing heat waves in the 21st century. Science, v. 305, p. 994-997, 2004.

MISHRA, V. et al. Changes in observed climate extremes in global urban areas. Environmental Research Letters, v. 10, n. 2, 024005, 2015.

QUEIROZ ROSENDO, E. E. Q. et al. Extreme rainfall and territorial disorder in the construction of risk: a case study in the Cabaceiras Municipality of Paraíba, Brazil. Cuadernos de Geografía - Revista Colombiana de Geografía, v. 24, n. 2, p. 189-203, 2015.

RUFINO, R. et al. Surtos de diarreia na região Nordeste do Brasil em 2013, segundo a mídia e sistemas de informação de saúde-Vigilância de situações 
climáticas de risco e emergências em saúde. Revista Ciência \& Saúde Coletiva, v. 21, n. 3, p. 777-788, 2016.

SCARROTT, C.; MACDONALD, A. A review of Extreme Value Threshold Estimation and uncertainty quantification. REVSTAT, v. 10, p. 33-60, 2012.

SENEVIRATNE, S. I. et al. Changes in climate extremes and their impacts on the natural physical environment. In: Managing the risks of extreme events and disasters to advance climate change adaptation, p. 109-230, 2012.

SILVA, M. A. M. da et al. Fatores socioambientais influenciados pela seca na conservação da Caatinga. HOLOS, v. 4, p. 245-257, 2016.

SOUZA, J. C. O. de. Análise do evento climático extremo ocorrido na Região Leste de Alagoas: bacias hidrográficas dos rios Mundaú e Paraíba do Meio. Revista Brasileira de Geografia Física, v. 4, n. 2, p. 377-395, 2011.

SOUSA, F. A. S. de.; SOUZA, J. T. A. Impactos da seca de 2012-2013 nas culturas agrícolas versus ações de convivência com a seca no município de ParariParaíba. Cadernos de Agroecologia, v. 10, n. 2, 2015.

SOUZA, P.; CAVALCANTI, I. F. A. Atmospheric centres of action associated with the Atlantic ITCZ position. International Journal of Climatology, v. 29, n. 14, p. 2091-2105, 2009.

TORRES R. R.; FERREIRA, N. J. Case Studies of Easterly Wave Disturbances over Northeast Brazil suing the Eta Model. Weather and Forecasting, v. 26, n. 2, p. 225-235, 2011.

TRENBERTH, K. E. Framing the way to relate climate extremes to climate change. Climatic Change, v. 115, p. 283-290, 2012.

WILLEMS, P. et al. Climate change impact assessment on urban rainfall extremes and urban drainage: methods and shortcomings. Atmospheric Research, v. 103, p. 106-118, 2012.

WORLD METEOROLOGICAL ORGANIZATION. Guidelines on Analysis of extremes in a changing climate in support of informed decisions for adaptation, 2009, 53 p.

Washington Luiz Félix Correia Filho - Possui Graduação em Meteorologia pela Universidade Federal de Alagoas. Mestrado em Meteorologia pela Universidade Federal de Campina Grande e Doutor em Ciências Climáticas na Universidade Federal do Rio Grande do Nortel.

Paulo Sérgio Lucio - Graduação em Matemática pela Universidade Federal do Espírito Santo. Mestrado em Estatística pela Universidade Estadual de Campinas. Possui Doutorado em Geofísica pelo Institut de Physique du Globe de Paris e Pós-Doutorado no Instituto Superior Técnico de Lisboa, no Centro de Geofísica da Universidade de Évora e no Laboratoire d'Océanographie et du Climat, Expérimentation et Approches Numériques (LOCEAN) na França. Atualmente é Professor Associado do Departamento de Ciências Atmosféricas e Climáticas da Universidade Federal do Rio Grande do Norte. 
Maria Helena Constantino Spyrides - Graduada em Estatística pela Universidade Federal do Rio Grande do Norte. Mestre em Agronomia (Estatística e Experimentação Agronômica) pela Universidade de São Paulo e Doutora em Saúde Pública pela Fundação Oswaldo Cruz. Atualmente é professora adjunta lotada no Departamento de Estatística da Universidade Federal do Rio Grande do Norte.

Contribuição de cada autor no desenvolvimento do artigo

Todos os autores ofereceram substanciais contribuições científicas e intelectuais ao estudo. As tarefas de concepção e design do estudo, preparação e redação do manuscrito, bem como a revisão crítica foram desenvolvidas em grupo. O primeiro autor ficou especialmente responsável pelo desenvolvimento teórico-conceitual, pela aquisição de dados e suas interpretações e análise; o segundo e terceiro autores contribuíram com a revisão teórico-conceitual e na discussão dos resultados obtidos.

Recebido para publicação em 07 de agosto de 2016

Aceito para publicação em 01 de outubro de 2016 